\title{
Linear Programming Models for Traffic Engineering in 100\% Survivable Networks under combined IS-IS/OSPF and MPLS-TE
}

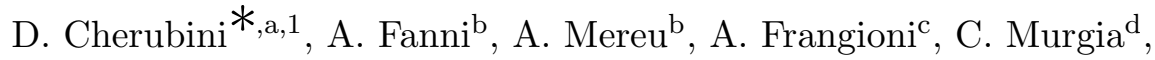 \\ M. G. Scutellàc, P. Zuddas ${ }^{\mathrm{e}}$ \\ ${ }^{a}$ Tiscali International Network, loc. "Sa Illetta", Cagliari, Italy \\ ${ }^{b}$ DIEE, Università di Cagliari, P.za d'Armi 1, Cagliari, Italy \\ ${ }^{c}$ Dipartimento di Informatica, Università di Pisa, Largo B. Pontecorvo 3, Italy \\ ${ }^{d}$ Tiscali Italia S.p.A., loc. "Sa Illetta", Cagliari, Italy \\ ${ }^{e}$ DIT, Università di Cagliari, P.za d'Armi 1, Cagliari, Italy
}

\begin{abstract}
This paper concerns the problem of minimizing the maximum link utilization of IP telecommunication networks under the joint use of traditional IGP routing protocols, such as IS-IS and OSPF, and the more sophisticated MPLS-TE technology. It is shown that the problem of choosing the optimal routing, both under working conditions and under single link failure scenarios, can be casted as a Linear Program of reasonable size. The proposed model is validated by a computational experimentation performed on synthetic and real networks: the obtained results show that the new approach considerably reduces the maximum link utilization of the network with respect to simply optimizing the IGP weights, at the cost of adding a limited number of label switched paths (LSPs). Optimizing the set of IGP weights within the overall approach further improves performances. The computational time needed to solve the models matches well with real-time requirements, and makes it possible to consider network design problems.
\end{abstract}

Key words: Routing problems, LP models, Robustness.

\footnotetext{
${ }^{*}$ Corresponding author

Email addresses: davide.cherubini@alcatel-lucent.com (D. Cherubini), fanni@diee.unica.it (A. Fanni), anna.mereu@diee.unica.it (A. Mereu), frangio@di.unipi.it (A. Frangioni), cmurgia@tiscali.com (C. Murgia), scut@di.unipi.it (M. G. Scutellà), zuddas@unica.it (P. Zuddas)

${ }^{1}$ D. Cherubini is now with Alcatel-Lucent Bell Labs Ireland, Blanchardstown Ind. Park, Blanchardstown, Ireland.
} 


\section{Introduction}

Communication and computer networks have high deployment and maintenance costs, which makes development of optimization models for their design and routing an important subject to both researchers and practitioners [1, 2]. This is even more true as the continuous development of new technologies keeps offering new avenues for optimization, while requiring to re-think established operations. Several different forms of network design problems exist depending on the specific details of the network under consideration: the layer (physical, IP, ... ), the corresponding routing protocols, the routing and deployment costs, and the survivability requirements specifying under which set of conditions the network should be guaranteed to remain operational. Whilst very general forms of design and routing problems can be devised [3], being able to solve them within the time limits dictated by the application environments crucially depends on exploiting the specific technological characteristics of the network at hand. Many of these problems are hard to solve, both in theory and in practice. For instance, optimal routing under the available IGP (Interior Gateway Protocol) protocol, such as Intermediate System to Intermediate System (IS-IS) or Open Shortest Path First (OSPF), requires finding a set of weight links (integers in the range $\left[1,2^{24}\right]$ ) that completely determine the routing of the demands; even with no survivability assumptions this is a $\mathcal{N} \mathcal{P}$-hard problem that cannot be approximated with a factor better than $3 / 2$ [4]. Thus, even simple routing on current IP networks is a difficult problem; fortunately, new Traffic Engineering (TE) techniques have been developed to enable Internet Service Providers (ISP) to route the traffic along the network. In particular, Multi-Protocol Label Switching (MPLS-TE) networks enable ISPs to move traffic from congested links to less loaded areas of the network setting up constrained Label Switched Paths (LSPs). However, the use of a "full mesh" MPLS-TE, where each origin-destination pair gets its dedicated LSP (possibly more than one to take into account different kinds of traffic) is not scalable, since it typically requires $O\left(n^{2}\right)$ paths which is not considered to be practical. Hence, one should rather search for an optimal set of complementary LSP tunnels to be used in combination with the IGP protocol.

This is especially important as, besides optimizing network resources utilization in the nominal case, these techniques can be used to implement network survivability techniques which allow resilience to equipment failure or congestion. Several different optimization models dealing with different 
forms of survivability have been developed [5, 6, 7, 8, 9, 10, 11, 12, 13, 14, 15, 16, 17, 18; in this paper we focus on single link failures, which represent by far the most common form of unplanned failure in real networks [19]. To survive a (single-link) failure, restoration routing is performed; this can be path (end-to-end) or link (local) restoration [20]. In the former scheme, if a failure is present along a path, the source node detects it and activates backup paths; due to its global nature, this may cause very large restoration latencies. In the link restoration scheme, a backup path is found to protect the failed link, rather than the entire path; although this may use more resources, it is attractive in that it can meet strict restoration latency requirements.

Furthermore, the local restoration scheme fits very well with a specific combination of network technology as far as the resulting optimization models are concerned. In particular, we will show that if restoration paths are all chosen by the IS-IS/OSPF protocol, for a fixed set of weight links the survivable routing problem using a combination of the available routing technologies (IS-IS/OSPF and MPLS-TE) can be modeled as a relatively smallsize Linear Program (LP). While the assumption of fixed weight links may appear to be a rather strong one, our computational results show that the model provides very good resources utilization with any reasonably chosen set of IS-IS weights, while dictating the use of a small set of LSP tunnels and requiring a comparatively short running time to be solved by off-the-shelf methods. This is somewhat surprising, as several routing problems under realistic conditions are difficult, especially if survivability has to be taken into account, as discussed in Section 3. Indeed, while several optimization models under the MPLS-TE technology have been presented in the literature $[20,21,22,23,24,25,26$, the majority of these papers either only consider the normal (or working) condition, or deal with survivability constraints as a two-phase design process, where the working paths are firstly chosen to optimize the routing in working conditions, and only then restoration (or backup) paths are selected that protect the links along the primary LSP. To the best of our knowledge, no paper considers at the same time optimal routing and survivability in the specific (but very reasonable in practice) set of technological requirements described here, which fortunately happen to make it easy to solve. This is interesting in that the efficient solution of the routing problem is usually a crucial step in the far more complex network design problems in telecommunication networks [27, 9, 2, 1].

The outline of the paper is as follows. Section 2 first presents some relevant backgrounds on various aspects of routing problems, then summarizes 
related works dealing with IGP and MPLS-TE optimization approaches. In Section 4 we describe the routing optimization models, while in Section 5 we demonstrate the suitability of the proposed optimization models by tests and comparisons on synthetic and real networks. Finally, Section 6 presents our conclusions and directions for future research.

\section{Background}

\subsection{Routing protocols}

IP backbone networks employ IGP routing protocols, such as IS-IS and OSPF, in order to compute the routes from each origin node to each destination node of the traffic demands. This is done by assigning a suitable metric value to each link of the network, and then routing along the shortest paths w.r.t. the selected metrics (weights). When Equivalent Cost Multiple Paths (ECMP) are enabled, the traffic flow is equally split among the alternative shortest paths linking each origin-destination pair. Generally, the traffic splitting mechanism is per packet round robin, where each packet matching a given destination is forwarded toward the corresponding egress node using the least recently used equal cost path.

The introduction of the Multi-Protocol Label Switching Traffic Engineering (MPLS-TE) technology has improved the dataflow management due to the traditional routing protocols. In fact, unlike IGP routing protocols, MPLS-TE networks can support destination-based and explicit routing simultaneously. Furthermore, MPLS-TE provides better handling of congestion and failures by suppling mechanisms to quickly find an alternate path if the primary path is no longer available. This Fast Re-Route (FRR) capability is critical for allowing service providers to offer high availability and high revenue Service Level Agreements. However, FRR requires that the operator explicitly sets secondary paths for all the possible failure scenarios, which can be excessively time consuming; besides, choosing appropriate reserve paths is nontrivial. An interesting alternative is therefore to rely on the link restoration approach, which is technically obtained by using "Implicit LSP" as reserve paths; this causes the commodities affected by the fault to be re-routed using the IGP (IS-IS/OSPF), thereby negating the need to explicitly choosing the reserve paths.

\section{Related works}

Several strands of research are related to the results in this paper. 
The first concerns optimal routing using IS-IS/OSPF protocols. This can be cast in different ways [28], and several (mostly heuristic) approaches have been proposed to solve it such as Tabu Search (TS) [29], genetic algorithms [30, 31] and tailored heuristics [32, 33]. An extended comparison of different heuristic approaches, comprising local search, Simulated Annealing (SA) and Lagrangian Relaxation, is presented in [34, both for the case where ECMP are allowed and for that where only a unique path for demand is allowed. The results of these papers show that optimal IS-IS/OSPF metrics not only are cumbersome to find, but also do not perform as well as an optimal MPLS-TE routing.

Other papers have addressed the problem of determining an optimal MPLS routing according to different objective functions. In particular, in 22] a TS algorithm is proposed to find a layout of MPLS-TE paths with the minimum number of hops, whereas in 23. the MPLS routing problem is modeled in terms of an off-line multiobjective Mixed Integer Linear Programming (MILP) model that looks for the best trade-off between the minimal routing delay, the optimal load balancing, and the minimal splitting of traffic trunks. In [35] and in [36] a multipath adaptive traffic engineering mechanism is introduced which aims at avoiding the network congestion by adaptively balancing the load among multiple paths based on measurements and analysis of the path level. In [37] and 38 an online traffic engineering approach is proposed which aims to determine minimum interference LSP tunnels, defined as paths that maximize the weighted sum of flows between all the other node pairs (called WSUM-MAX problem): a heuristic online procedure is proposed which allows a good LSP acceptance rate and re-routing performance, thus limiting the well known online traffic engineering drawbacks related to unavailability of bandwidth at the request time.

Relatively few papers deal with combined IGP/MPLS routing, and most of these employ a two phase (hierarchical) approach by optimizing separately the two technologies. In [39] the routing problem is formulated as a service differentiated model where the IP flows are classified (based on experience) into low bandwidth demanding (LBD) and high bandwidth demanding (HBD) flows at the ingress of the network; LBD flows are routed along the IGP shortest paths, while HBD flows over bandwidth-guaranteed LSP tunnels. The optimization problem seeks the best network configuration that maximizes bandwidth usage (optimality), minimizing rerouting upon failure (reliability) and reducing the signaling overheads resulting from a full IP tunneling (scalability); several simulations are required to choose 
the value of the calibration parameters in the objective function expressing the trade-off among optimality, reliability and scalability. In 24] IGP optimization is firstly performed using SA, and then a set of complementary LSP paths are computed to improve the IGP solution using a MILP model; the results show that the use of LSPs greatly improve the network utilization. In 25] the basic idea of this hybrid approach is used to combine both the simplicity and robustness of IGP routing and the flexibility of MPLS-TE: SA is used to select a small number of LSPs under different objective functions so as to be independent is IGP weights configuration. In [26] an open source toolbox for traffic engineering methods (TOTEM) is presented which provides facilities for IS-IS/OSPF metrics optimization, primary and backup LSP routing, and BGP (Border Gateway Protocol) simulations [40]. A mathematical programming formulation for the routing problem under the combined IGP/MPLS scenarios is presented in [41] which is solved with a heuristic decomposition approach employing evolutionary algorithm and simulated allocation. In [42] three different strategies to implement the coexistence of the IGP and MPLS protocols have been presented: the idea is to add only a limited number of LSP tunnels under conditions on the maximum number of hops and maximum delay. A comparison among the three approaches is presented in [43]: the simulations demonstrate that each of the three models allows to considerably reduce the congestion level of the network while keeping the delay and hop-count metrics within an acceptable range. Finally, in [44] the traffic engineering problem has been formulated as a LP with hybrid MPLS/OSPF routing, avoiding the drawbacks of both the OSPF weight optimization, which triggers network-wide convergence and significant traffic shift, and those of pure MPLS approach, which requires a full mesh of tunnels to be configured throughout the network.

None of the aforementioned papers considers the survivability issue embedded in the overall optimization process. In the majority of the literature, survivability is addressed by simulation and/or heuristic approaches. For ISIS/OSPF networks, survivability has been addressed in [29, 45, 46, 47, 27]. In particular, in [29, 45, 47] TS approaches are used in order to determine robust metrics that guarantee survivability both in working condition and in all single link failure scenarios. In [27, the same problem has been solved using an evolutionary algorithm, addressing also the failure of the routers. In [46, a bicriteria algorithm has been used, where the working condition and failure scenarios are separately considered; basically, the authors use a local search algorithm based on TS where the objective function "drives" the search toward Pareto optimal solutions. All the authors report a significant 
Table 1: Summary of Traffic Engineering Techniques in IP networks

\begin{tabular}{|c|c|c|}
\hline technique & no survivability & survivability \\
\hline IGP weight optimization & {$[28,[30,31,32,33,34]$} & {$[29,[45$, 46, 47] $]$} \\
\hline MPLS optimization & {$[37,[38,[22,[23,[28]$} & {$[20,21]$} \\
\hline Hybrid IGP/MPLS & {$[24,[42,[43]$} & {$[50][$ Present Paper] } \\
\hline
\end{tabular}

reduction of the link congestion in case of single link failure, at the price of a higher maximum occupation in working conditions; furthermore, the IS-IS/OSPF weight adjustment induces potentially large traffic shift in the network, which may cause service degradation. Due to these reasons, changing link weights can only be done infrequently, and therefore the required computational time, although high, can be disregarded when considering the overall time of the presented approaches. Survivability under the MPLS-TE technology has been formulated in [48] in terms of the Spare Capacity Allocation (SCA) problem. The idea is to mitigate the impact of a single link failure by allocating spare capacity on the links and creating arc-disjoint LSP backup paths for each primary LSP. This goal can be unachievable for pathological network topologies [49]. In [21] the SCA problem is addressed by separate design of working and restoration paths resulting in a minimum capacity usage; for this purpose, two LP models are sequentially solved. An improvement of the model is developed to take into account load balancing. In [20], the problem of distributed routing of restoration paths is addressed by introducing the concept of backtracking to bound the restoration latency; a two steps heuristic algorithm is proposed where primary paths and backup paths are computed in two separated steps.

All the above approaches obtain optimal solutions of simple optimization problems, or sub-optimal solutions of wider problems. Very few approaches address combined IS-IS/OSPF and MPLS-TE routing and single link failures simultaneously. In [50, some of the authors presented preliminary results of a combined routing approach: a LP model jointly uses the IGP routing protocols and the MPLS-TE technology to minimize the maximum link utilization under single-link survivability constraints and the path restoration scheme. This leads to significantly more complex models w.r.t. those presented here for the link restoration scheme. A summary of the literature overview on Traffic Engineering techniques in IP networks is reported in Table 1 .

\section{The link restoration combined routing problem}

Let $\mathcal{G}=(\mathcal{N}, \mathcal{E})$ be an undirected graph modeling a telecommunication network, where $\mathcal{N}(|\mathcal{N}|=n)$ is the set of nodes (routers) and $\mathcal{E}(|\mathcal{E}|=\mu)$ 
is the set of edges (links) of the network. A bi-directional transmission capacity $c_{i j}$ is associated with each (undirected) link $\{i, j\}$, i.e., one can simultaneously transmit up to $c_{i j}$ information from $i$ to $j$ and $c_{i j}$ information from $j$ to $i$ without the two transmissions interfering with each other. In the following we will often use the bi-direction of $\mathcal{G}$, i.e., the directed graph $\mathcal{G}^{\prime}=(\mathcal{N}, \mathcal{A})$ having the same set of nodes of $\mathcal{G}$, whereas $\mathcal{A}(|\mathcal{A}|=m=2 \mu)$ is the set of pairs of directed $\operatorname{arcs}(i, j)$ and $(j, i)$ for each edge $\{i, j\} \in \mathcal{E}$. We will often employ the short-hand notation $l$ for denoting an edge $\{i, j\} \in \mathcal{E}$, with the two corresponding directed arcs denoted by $l_{+}=(i, j)$ and $l_{-}=$ $(j, i)$, respectively. We will also denote by $F S(i)$ and $B S(i)$, respectively, the standard forward star and backward star of a generic node $i$ in $\mathcal{G}^{\prime}$.

In general, one has a set $\mathcal{F}\left(|\mathcal{F}|=k\right.$, and usually $\left.k \in O\left(n^{2}\right)\right)$ of origindestination pairs, or commodities; for each $f \in \mathcal{F}, s(f), t(f)$ and $d^{f}$ denote respectively the origin node, the destination node and the traffic demand of the commodity $f$. For each $f \in F$ and $(i, j) \in \mathcal{A}$ one also has the rational number $x_{i j}^{f} \in[0,1]$ representing the fraction of flow of the commodity $f$ that is routed by IS-IS/OSPF along the arc $(i, j)$; we assume that this routing data (succinctly denoted by the $m \times k$ routing matrix $\mathcal{X}$ ) is given as input, having been computed with a suitable set of IS-IS/OSPF metrics, e.g. obtained with the approaches proposed in [47, 28, 29]. One could construct the model using the "natural" commodities $\mathcal{F}$; this would be required if there were e.g. commodity-dependent costs or bounds. However, since this is not the case in our applications, it is preferable to consider the aggregated notion of commodity, whereby all commodities having the same ingress node are considered to be "the same kind of flow". In order to do that we need some further notation: $\mathcal{H}$ is the set of the origin nodes, which therefore coincides with the set of aggregated commodities, and for each node $v \in \mathcal{H}$ the set $\mathcal{F}(v)=\{f \in \mathcal{F}: s(f)=v\}$ contains the commodities having $v$ as their ingress node.

\subsection{Nominal model}

We start by presenting the nominal model, i.e., without survivability constraints. Given $\mathcal{G}, \mathcal{F}$ and $\mathcal{X}$, the problem of satisfying the demands of the commodities in $\mathcal{F}$ exploiting both IS-IS/OSPF and MPLS-TE routing technologies, in such a way as to minimize the maximum arc utilization of the network, can be cast as the following compact LP.

$$
\sum_{f \in \mathcal{F}}^{\min } u_{\max }^{f} x_{i j}^{f}+\sum_{v \in \mathcal{H}} w_{i j}^{v} \leq u_{\max } c_{i j} \quad(i, j) \in \mathcal{A}
$$




$$
\begin{aligned}
& \sum_{j \in B S(i)} w_{j i}^{v}-\sum_{j \in F S(i)} w_{i j}^{v}=\left\{\begin{array}{lll}
\sum_{f \in \mathcal{F}(v)} i s^{f}-d^{f} & \text { if } i=v \\
d^{f}-i s^{f} & \text { if } i=t(f) & i \in \mathcal{N} \\
0 & f \in \mathcal{F}(v) & v \in \mathcal{H}
\end{array}\right. \\
& 0 \leq w_{i j}^{v} \\
& 0 \leq i s^{f} \leq d^{f} \\
& (i, j) \in \mathcal{A}, v \in \mathcal{H} \\
& f \in \mathcal{F}
\end{aligned}
$$

The single variable $u_{\max }$ represents the maximum utilization over all the arcs of the network, the variables $i s^{f}$ represent the share of traffic demand $d^{f}$ of commodity $f$ carried by IS-IS/OSPF, while the variables $w_{i j}^{v}$ represent the share of traffic demand of the aggregated commodity $v$ carried by LSP along arc $(i, j)$ via the MPLS-TE protocol. The objective function (1) measures the maximum arc utilization, which has to be minimized; as shown in [51, and validated with the results in the present paper, minimizing the maximum link utilization tends to maximize the available bandwidth, thus balancing the load in the network as a side effect. Constraints (2) ensure that $u_{\max }$ is an upper bound on the utilization of each arc, measured as the ratio between the traffic routed along it via the IS-IS/OSPF protocol and the MPLS-TE technology (first and second term of the left-hand-side, respectively) and its capacity. Constraints (3) ensure that the communication demand of each commodity is entirely satisfied, by suitably combining the traffic sent via IS-IS/OSPF and that sent via MPLS-TE. Note that if every commodity $f$ could only be routed either by IS-IS/OSPF or by MPLS-TE, then $i s^{f}$ should be defined as binary, thus turning the problem into a (much more difficult to solve) MILP. Fortunately, current routers operations allow to direct to an MPLS-TE path every chosen fraction of a given commodity, thereby permitting the recourse to a more computationally tractable LP formulation.

This is a quite classical (aggregated) node-arc multicommodity flow formulation [49], common to very many network routing and design problems [28, 1], augmented with the extra terms necessary to take into account the possibility of routing along the (fixed) IS-IS/OSPF paths. It would be easy to develop not only disaggregated versions of the model, which may be useful for either modeling or algorithmic reasons [52, but also versions using the arc-path formulation, which lends itself nicely to (stabilized) column generation techniques that can considerably speed-up the solution of the models [53. This is actually necessary when path restoration is used instead of link restoration, even in the same combined IGP/MPLS setting [50]. However, 
for the current set of technological assumptions the above compact node-arc formulation is sufficient; due to its relatively small size $(h m+k+1$ variables and $m+h n$ constraints, where $h=|\mathcal{H}| \leq n)$, it can be solved quickly by off-the-shelf LP components.

Using the proposed model in an actual network management setting requires the following steps:

1. computation of a proper set of IS-IS/OSPF routing metrics and the corresponding IS-IS routing, represented by matrix $\mathcal{X}$;

2. solution of the model to obtain a complementary set of LSP paths;

3. setup of the explicit LSPs determined by the model.

All these steps can be easily automatized. Actually, one could even setup an iterative process, whereby after step 2. the MPLS flows $w$ dictated by the model are "fixed" (subtracted from the demands and arc capacities), and step 1. is repeated in order to adapt the metrics to the chosen set of LSP tunnels. This may be repeated as long as improvements are obtained, thus providing a convenient block-descent (heuristic) approach to the problem of simultaneously choosing the metrics and the complimentary set of LSP paths. Yet, in all tested instances even the first re-computation of the metrics has never improved the solution once, so it appears that improving on the solution obtained by the simple "optimize metrics first, choose LSPs second" approach is not easy.

Furthermore, note that step 3. requires the decomposition of the obtained flow solution $w$ into paths. This is quickly obtained by the path decomposition algorithm [49]; unfortunately, the decomposition is in general not unique. Thus, the optimal solution of (1) - (5) may be decomposed in different ways, ultimately leading to a different number of LSP paths (the same would be true for a disaggregated formulation). Finding the decomposition which minimizes the number of paths is not an option, as this is a $\mathcal{N} \mathcal{P}$-Hard problem [54. Actually, this is not the only (and possibly not even the main) reason why model (1) - (5) does not allow any direct control on the final number of LSP paths: indeed, as shown by the following proposition, its optimal solutions set almost surely is highly degenerate.

Proposition 4.1. Each instance of (1) - (5) has an optimal solution where $i s^{f}=0$ for all $f \in \mathcal{F}$.

Proof. For any $f \in \mathcal{F}$, the flow routed on each arc $(i, j)$ by the IS-IS/OSPF protocol for the commodity $f$, given by $i s^{f} x_{i j}^{f}$, can be represented in terms 
of the flow variables $w_{i j}^{v}$. Therefore, for any feasible solution, another feasible solution exists where the share $i s^{f}$ of the demand routed by the IS-IS protocol is routed by the MPLS-TE protocol: both solutions produce the same link utilization, and hence the same value of the objective function.

Thus, the above model does not control the total number of the resulting LSPs; a solution can always be obtained that does not use the IS-IS/OSPFonly routing at all, possibly at the cost of setting a very large number of explicit LSP paths. While having a solution with many LSPs may not have a large operational cost, it can be argued that, for the same value of the maximum network utilization, a solution using a smaller number of LSP paths could be preferred. Explicitly minimizing the number of employed LSP leads to a difficult problem; however, one can obtain good results in practice by replacing (1) with

$$
u_{\max }+\delta \sum_{v \in \mathcal{H}} \sum_{(v, j) \in F S(v)} w_{v j}^{v}
$$

where $\delta$ is a "small" value. This penalizes each unit of flow leaving the source nodes, thereby ensuring that LSP paths are used only if they strictly decrease the maximum utilization level. This may reduce the number of the LSPs, as shown in 5.4. Note, however, that while a reduction is most often experienced in practice, there is no guarantee that the number of the LSPs will actually decrease. Indeed, while any $\delta>0$ allows one to reduce the total amount of flow which is globally sent through the LSPs (w.r.t. the solution obtained by setting $\delta=0$ ), the reduced amount of flow may split up in different ways, ultimately along a larger number of LSPs. This is consistent with the fact that even splitting a given flow into the minimum number of paths is a hard problem [54]. Anyway, since the solution time of the model is very low, solving it repeatedly with different values of $\delta$ (among which $\delta=0$ ) and picking the solution with the lowest number of LSPs (among all with the minimum value of $u_{\max }$ ) can be a convenient heuristic to try to reduce the number of LSPs.

\subsection{Survivable model}

We now extend the previous model in order to consider survivability. We consider single link failures and define $\mathcal{L} \subseteq \mathcal{E}$ as the set of all possible failed links, or, equivalently, the set of the failure scenarios. We therefore refer to each element $l \in \mathcal{L}$ both as a failed link and as a failure scenario. The model can be easily extended to multiple link failures and/or node (router) failures, possibly at the cost of a substantial increase in size. Each single link failure scenario will be addressed under the link restoration mechanism, 
whose backup paths are realized in terms of implicit LSPs. Recall that the operational advantage of implicit LSPs is that they are automatically determined using the conventional IS-IS/OSPF metrics and without any intervention, whereas in case of an explicit LSP each backup path has to be explicitly specified by the network operator.

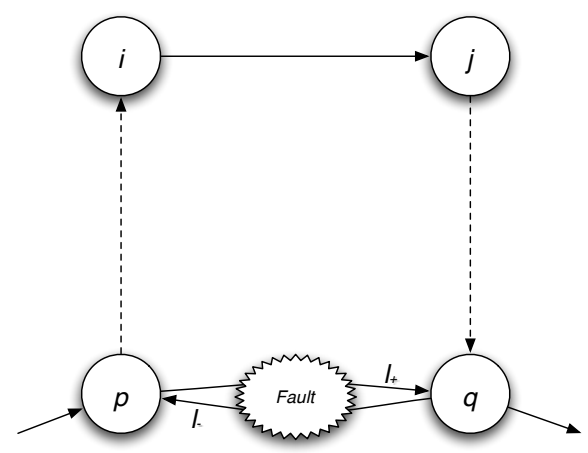

(a) Rerouting flow $p \rightarrow q$

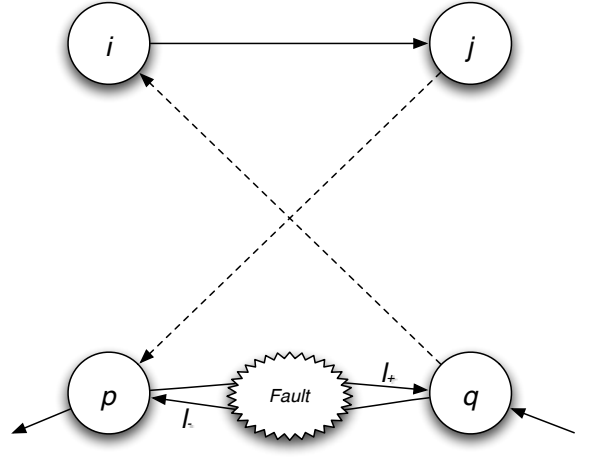

(b) Rerouting flow $q \rightarrow p$

Figure 1: Rerouting flows in case of edge failure

For each failure scenario $l$, a $(m-2) \times k$ routing matrix $\mathcal{X}^{l}$ is defined, whose element $x_{i j}^{f, l}$ is the fraction of the flow of commodity $f$ that is routed by IS-IS/OSPF protocol along the arc $(i, j)$, when the link $l$ fails (note that a link failing means that both arcs representing the link traversed in the two possible directions fail). Each of these routing matrices $\mathcal{X}^{l}$ can be easily determined, prior to solving the model, given the input set of IS-IS/OSPF metrics, by computing shortest paths over a graph obtained from $\mathcal{G}^{\prime}$ by removing the link $l$, i.e., by removing both $l_{+}$and $l_{-}$. Thus, the routing matrices $\mathcal{X}^{l}$ are input data. We also define $x_{i j}^{l_{+}, l}$ and $x_{i j}^{l_{-}, l}$ as the fraction of the MPLS-TE traffic, routed along the arcs $l_{+}$and $l_{-}$, respectively, under the nominal working conditions, that in case of failure of the link $l$ are rerouted along the arc $(i, j)$, via the IS-IS/OSPF protocol, according to the link restoration strategy. Note that the rows of the routing matrices correspond to the arcs, and the columns correspond to the commodities. Hence, $x_{i j}^{l_{+}, l}$ is the element of the routing matrix $\mathcal{X}^{l}$ corresponding to the arc $(i, j)$ and the commodity $f_{+}=(p, q)$, where $p$ and $q$ are the starting and ending nodes of the directed arc $l_{+}$as well as the origin and destination nodes of the commodity $f_{+}$. Similarly, $x_{i j}^{l_{-}, l}$ is the element of the routing matrix $\mathcal{X}^{l}$ corresponding to the arc $(i, j)$ and the commodity $f_{-}=(q, p)$, where $q$ and $p$ are the starting and ending nodes of the directed arc $l_{-}$as well as the origin and destination nodes of the commodity $f_{-}$. The commodities $f_{+}$and $f_{-}$have to be created unless they already belong to $\mathcal{F}$. 
With this data available, considering failure conditions in the model only corresponds to adding the following $|\mathcal{L}|(m-2)$ survivability constraints

$$
\sum_{f \in \mathcal{F}} x_{i j}^{f, l} i s^{f}+\sum_{v \in \mathcal{H}} w_{i j}^{v}+\sum_{v \in \mathcal{H}}\left(x_{i j}^{l_{+}, l} w_{l_{+}}^{v}+x_{i j}^{l_{-}, l} w_{l_{-}}^{v}\right) \leq u_{\max } c_{i j}
$$

for all $l \in \mathcal{L}$ and $(i, j) \in \mathcal{A} \backslash\left\{l^{+}, l^{-}\right\}$. The meaning of constraints (7) is pictorially illustrated in Fig. 1. The failure of the (undirected) link $l=$ $\{p, q\} \in \mathcal{L}$ means that the entire flow traversing the link in both directions, represented in the model by the two (directed) $\operatorname{arcs} l_{+}=(p, q)$ and $l_{-}=$ $(q, p)$, needs to be rerouted. The link restoration protocol thus re-routes all the flow traversing $l_{+}=(p, q)$ using the routes defined for the commodity $f_{+}=(p, q)$, and, similarly, re-routes the flow traversing $l_{-}=(q, p)$ using the routes defined for the commodity $f_{-}=(q, p)$. Clearly, the survivability constraints (7) are strictly dependent on the specific re-routing technology (link restoration). In particular, once a link fails, the entire flow along that link is re-routed like a commodity that has origin and destination in the two routers at the extremes of the failed link. The routing is performed using precomputed paths only dependent on the IS-IS/OSPF metrics. A consequence is that Proposition (4.1) does not necessarily hold for the survivable model. In fact, in case of failure of link $l$, each arc $(i, j)$ may behave differently depending on its usage according to the IS-IS/OSPF protocol or the MPLSTE technology. In the model this is expressed in terms of the coefficients $x_{i j}^{f, l}$ for the traffic sent via the IS-IS/OSPF protocol, or in terms of the coefficients $x_{i j}^{l_{+}, l}$ and $x_{i j}^{l_{-}, l}$ for the traffic which was routed via the MPLS-TE technology in normal condition, and which will be routed via IS-IS/OSPF protocol in case of failure of link $l$. Thus, IS-IS/OSPF and MPLS-TE may have a different impact on the arc utilization.

\section{Computational results}

The effectiveness of the proposed models has been tested on five different telecommunication networks: one of them is a synthetic network, two of them are real backbone networks, and the other two are test networks instances available in the literature. For all instances, at least two different sets of IGP weights are considered: default values suggested by the major routers vendors (all ones, i.e., the shortest paths are the paths with the minimum number of hops), and optimized metrics obtained by the TS algorithm of [47, which considers the failure scenarios in the search process. When available, the actual metrics used by the network operators have also been considered. Except when otherwise stated, the survivable model takes 
Table 2: Characteristics of the test instances

\begin{tabular}{|l|rrr|rrr|}
\hline network & $n$ & $m$ & $k$ & vars & ncnst & scnst \\
\hline random & 8 & 36 & 56 & 345 & 100 & 345 \\
IBCN & 37 & 114 & 1332 & 5551 & 1483 & 7867 \\
Tinet & 18 & 54 & 306 & 1279 & 378 & 1782 \\
Atlanta & 15 & 44 & 210 & 871 & 269 & 1193 \\
Géant & 23 & 76 & 445 & 2209 & 603 & 3267 \\
\hline
\end{tabular}

into account all possible link failures, i.e., $\mathcal{L}=\mathcal{E}$. The models have been coded in $\mathrm{C}++$, using the CLP solver under the OsiSolverInterface [55] to solve the LPs, and ran on a standard PC with a Pentium 4 processor. The reported computational times include the time required to find both the LSP decomposition and the IS-IS/OSPF routing matrix, but not the time to compute the optimized metrics by TS, since this is not supposed to be done frequently. All the results have been validated with the commercial simulation software OPNET Modeler [56].

\subsection{Test instances}

The main characteristics of the five test instances are described in Table 2 column "vars" reports the number of variables (which is the same in the two models), while columns "ncnst" and "sconst" report the number constraints for the nominal an survivable model, respectively.

The "random" network is a randomly generated synthetic network, whose topology and demand matrix are depicted in Figure 2. All links have a maximum capacity of $1000 \mathrm{Mbps}$, and the traffic matrix has been generated to produce congestion in case of single link failures.

Figure 2: Topology and traffic matrix of the random synthetic network

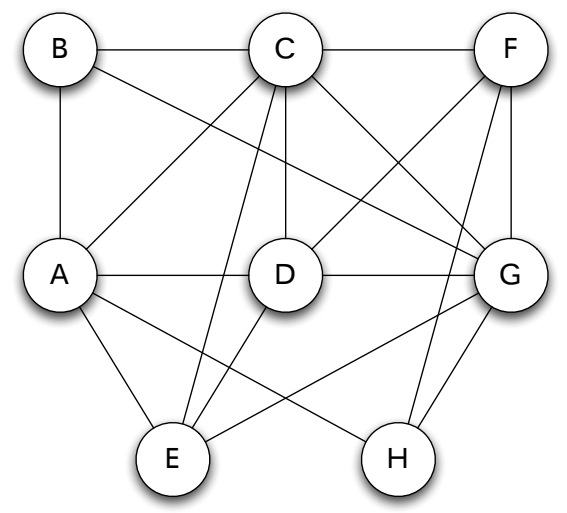

\begin{tabular}{|l|r|r|r|r|r|r|r|r|}
\hline & $\mathrm{A}$ & $\mathrm{B}$ & $\mathrm{C}$ & $\mathrm{D}$ & $\mathrm{E}$ & $\mathrm{F}$ & $\mathrm{G}$ & $\mathrm{H}$ \\
\hline $\mathrm{A}$ & & 40 & 30 & 60 & 20 & 10 & 70 & 70 \\
\hline $\mathrm{B}$ & 40 & & 60 & 20 & 70 & 40 & 50 & 50 \\
\hline $\mathrm{C}$ & 80 & 70 & & 60 & 40 & 80 & 10 & 60 \\
\hline $\mathrm{D}$ & 30 & 80 & 100 & & 40 & 60 & 70 & 120 \\
\hline $\mathrm{E}$ & 20 & 60 & 10 & 50 & & 20 & 60 & 30 \\
\hline $\mathrm{F}$ & 30 & 50 & 70 & 80 & 30 & & 100 & 40 \\
\hline $\mathrm{G}$ & 200 & 100 & 150 & 50 & 60 & 80 & & 60 \\
\hline $\mathrm{H}$ & 100 & 100 & 150 & 200 & 50 & 100 & 100 & \\
\hline
\end{tabular}

Both the "IBCN" network and the "Atlanta" network are part of the Zuse 
Institute Berlin's (ZIB) SNDlib [57], which is a library of test instances for network design. The former has been provided by the INTEC Broadband Communication Networks research group (IBCN) [58]; during the test, the capacity of the links has been fixed and the original demands values have been scaled in order to produce congestion over some links in case of single link failure, when the "default" metrics configuration is used. The latter is the "Atlanta D-B-M-N-C-A-N-N" instance; all links with zero capacity in the default configuration have been assigned a capacity of $1000 \mathrm{Mbps}$, while all other capacities have been multiplied by 10 .

The "Tinet" network is the Italian portion of Tiscali International Network (now Tinet). This is the only case when $\mathcal{L} \subset \mathcal{E}$, as failure of 4 specific links was guaranteed not to happen due to strategic decisions (uplink to the international backbone). Furthermore, for this instance we could test the IS-IS metrics actually used by the ISP. The whole Tiscali International Network logical topology can be found in [59]; however, the traffic matrices and the metrics used for the tests are confidential.

The "Géant" network is the actual backbone connecting European universities and research centers [60. Traffic matrices for this network have been provided in [61], sampled every 15 minutes for a period of almost 4 months. The traffic matrix used for our tests is IntraTM-2005-04-23-22-00.xml, corresponding to the $23^{r d}$ of April 2005 at 22:00 hour; it is a "worst case" matrix, since it has the highest level of mean and maximum link utilization.

\subsection{Computational results}

Computational results are summarized in Table 3 for all five instances. Column "metrics" indicates the IS-IS metrics employed: "default" are the unitary ones, "TS" are the optimized ones, and "Tinet" are these actually used by the ISP (for the Tinet network only). The obtained maximum arc utilization (in percentage) is reported both under "working" and "failure" condition, both for the case where only the IS-IS/OSPF routing protocol is used (which is equivalent to setting $i s^{f}=d^{f}$ for each $f$ in the model), and for the case where the combined IGP/MPLS approach is used. For the latter, column "\#LSP" also reports the number of LPSs dictated by the combined model for the survivable case. We do not report detailed running times, but these are uniformly quite low, ranging from less than one second (for both models) for the random network to around 200 and 400 seconds for the IBCN and the nominal and survivable models, respectively.

The results show that the combined approach can significantly improve the maximum link utilization w.r.t. the use of IS-IS/OSPF alone. However, the improvements are rather varied. The improvement can be as high as 
Table 3: Maximal arc utilization for all instances

\begin{tabular}{|l|c|rr|rrr|}
\multicolumn{2}{c}{ Table 3: Maximal arc utilization for all instances } \\
\cline { 3 - 7 } \multicolumn{2}{c|}{} & IS-IS/OSPF & \multicolumn{3}{c|}{ combined } \\
\hline \multirow{2}{*}{ network } & metrics & working & failure & working & failure & \# LSP \\
\hline \multirow{2}{*}{ IBCN } & default & 34 & 70 & 23 & 35 & 55 \\
& TS & 29 & 47 & 23 & 35 & 48 \\
\hline \multirow{3}{*}{ Tinet } & default & 71 & 101 & 42 & 75 & 671 \\
& TS & 55 & 74 & 41 & 65 & 585 \\
\hline \multirow{2}{*}{ Atlanta } & default & 72 & 128 & 59 & 83 & 134 \\
& Tinet & 66 & 117 & 59 & 83 & 83 \\
& TS & 61 & 85 & 59 & 83 & 139 \\
\hline \multirow{2}{*}{ Géant } & default & 33 & 142 & 22 & 110 & 129 \\
& TS & 32 & 83 & 22 & 83 & 147 \\
& default & 88 & 98 & 49 & 98 & 276 \\
& TS & 50 & 77 & 37 & 76 & 210 \\
\hline
\end{tabular}

$50 \%$ (random, failure) as well as zero (Atlanta and Géant, failure). The impact of metrics in the combined approach can be relevant (IBCN, Atlanta and Géant) or basically null (random and Tinet); when it is relevant, it can be so for the failure case alone (IBCN, Atlanta) or for both the working and the failure case (Géant). The combined model can improve for all sets of metrics and both in the working and failure case (random), or it can have next to no effect when the metrics are appropriately chosen (Tinet, Atlanta and Géant). The latter can happen only for the failure case (Atlanta and Géant), or both in the working and failure case (Tinet). It is, however, safe to say that (at least on the selected instances) the combined approach always improve (at least a little bit) in the working case and, most often than not, even in the failure case. Also, the improvement is quite significant, especially (in all cases but one: Géant failure) if one cannot or does not want to use optimized metrics.

However, the maximum arc utilization is not the only metric of interest for traffic engineers. We therefore report some results aimed at describing the traffic distribution properties of the combined approach more in detail. To do that, we draw in Figures 36 the utilization of each link, under working condition, for the IBCN, Tinet, Atlanta and Géant networks, respectively, for all the combinations of models and metrics. This pictures how evenly (or not) the traffic is spread across the network; the conventional wisdom is that the more even the distribution, the more the network is resilient to changes in the traffic pattern and failures. Similarly, we draw in Figures 7 10 the objective function value for each of the possible single link failures for the IBCN, Tinet, Atlanta and Géant networks, respectively, again for all the combinations of models and metrics. This is useful to show whether 
the routing succeeds in "evening out" the effect of failures irrespective of the failed links, which gives confidence in network resilience e.g. in the case of an unplanned-for second link failure, or some "hot spots" remain whose failure has a disproportionately large impact on congestion.

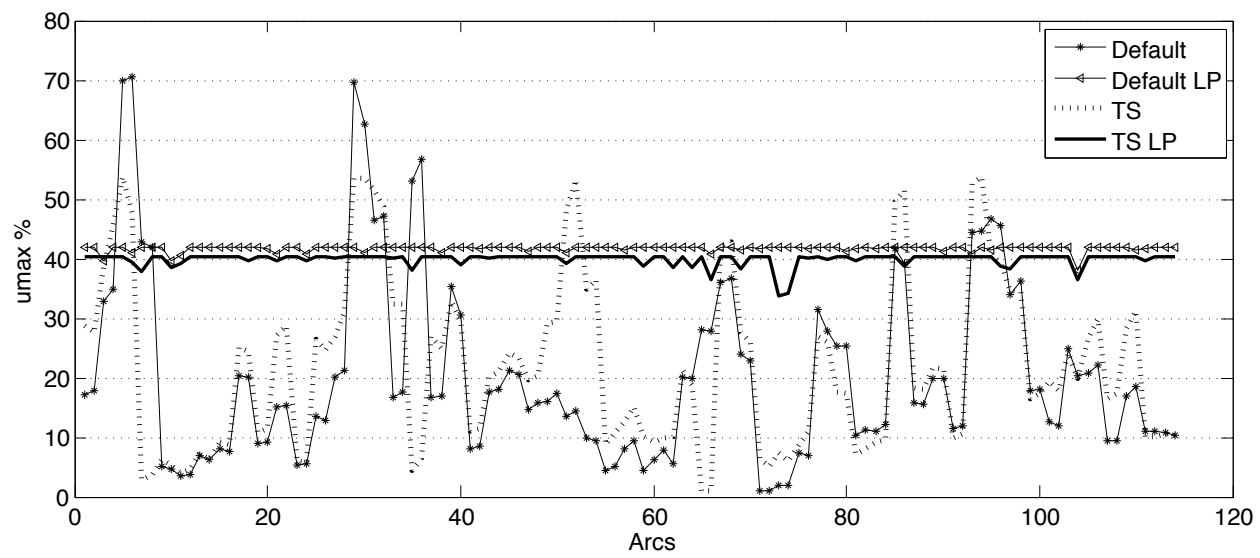

Figure 3: Link utilization under working condition for IBCN network

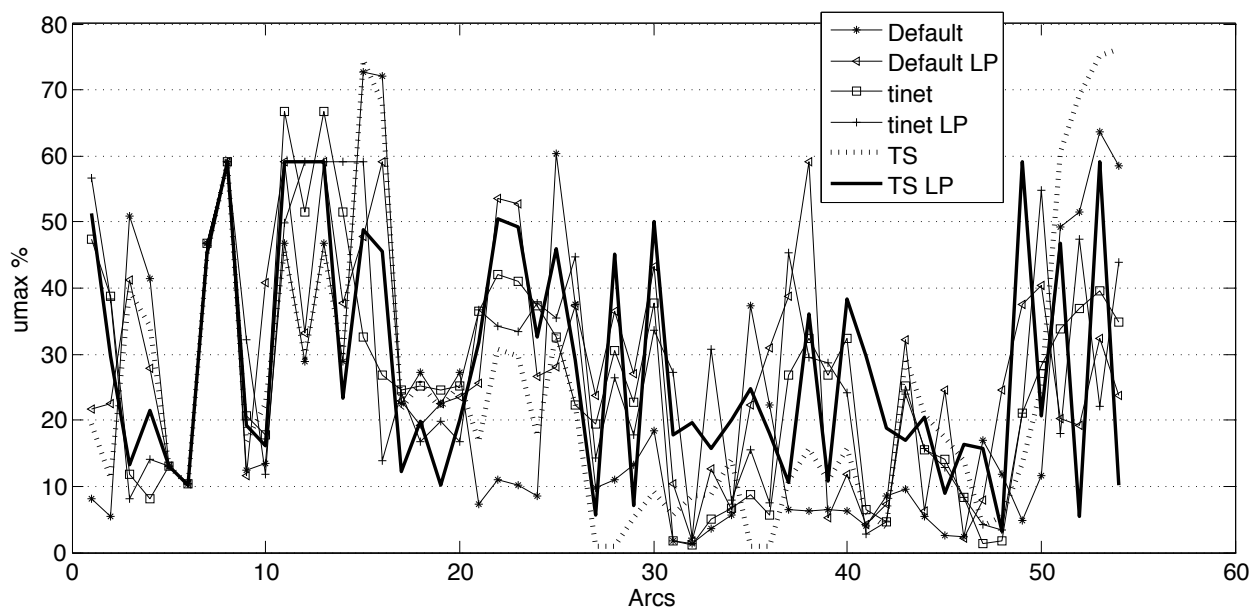

Figure 4: Link utilization under working condition for Tinet network

The figures show that, in general, the combined IGP/MPLS-TE routing is much more "even" than the IGP-only one. The effect is not always comparable: for the Tinet network (cf. Figure 4), while link utilization is indeed slightly lower and somewhat more evenly distributed with the combined routing schemes, the effect is by far less visible than in the other cases. Similarly, the combined routing significantly "smoothes" the network 


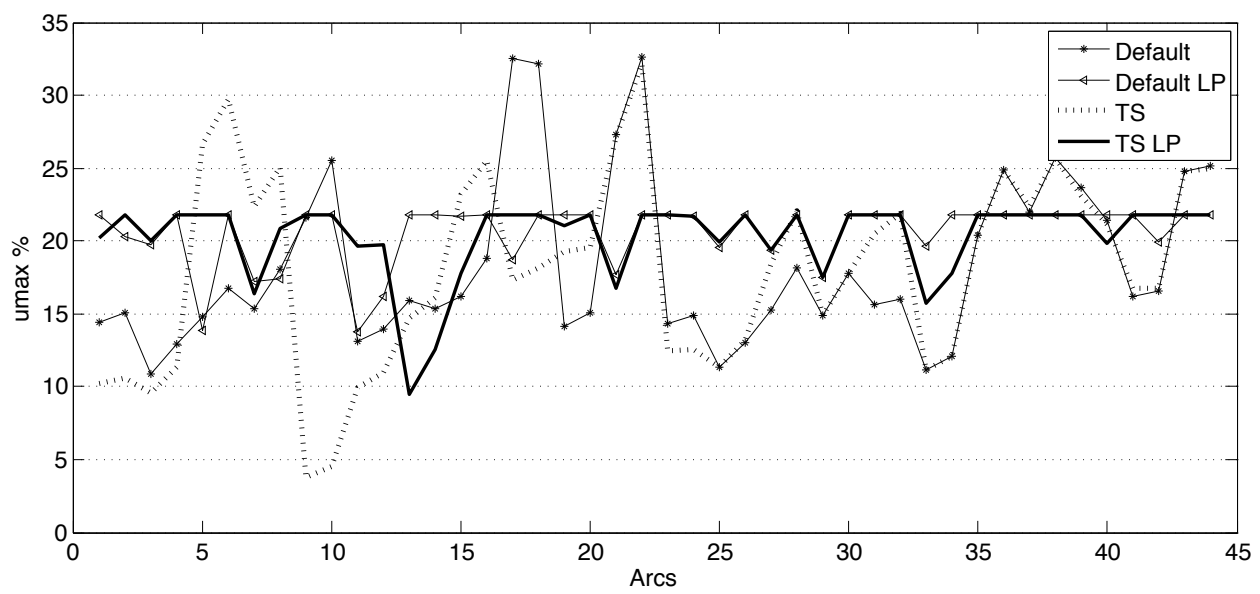

Figure 5: Link utilization under working condition for Atlanta network

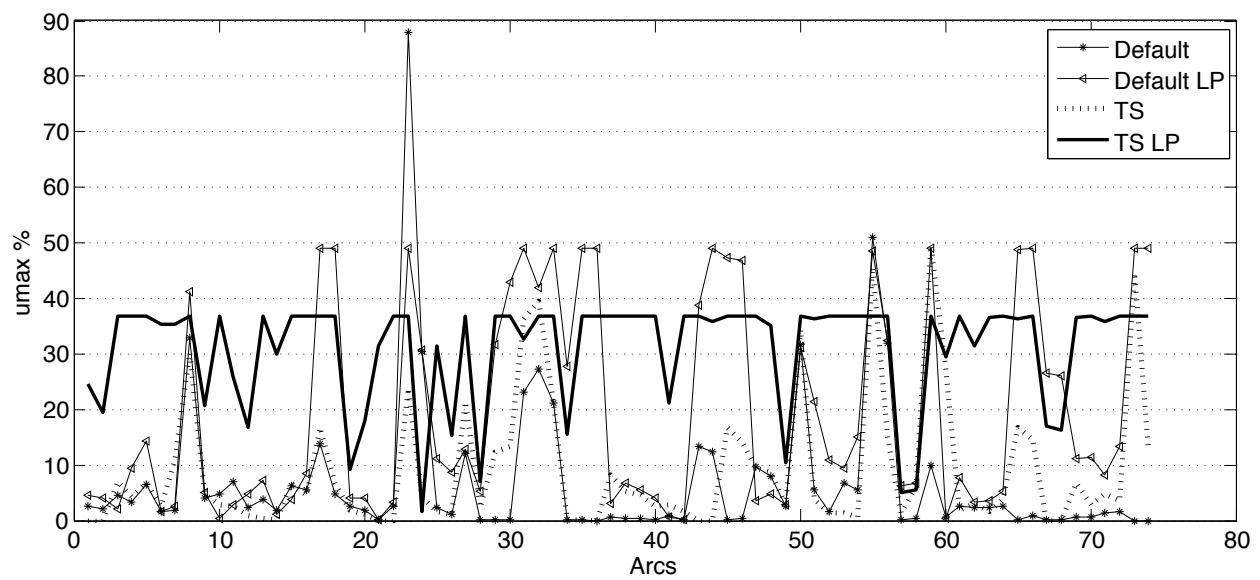

Figure 6: Link utilization under working condition for Géant network

response to single link failures. Again, the qualitative behavior of different networks can be different. For instance, while the combined routing allows to avoid congestion $\left(u_{\max }\right.$ greater than $\left.100 \%\right)$ for all metrics in both IBCN and Tinet, this is not true for Atlanta and Géant. In the latter, in particular, default metrics uniformly lead to "almost congestion" $\left(u_{\max }=98 \%\right)$ for each single failure. Yet, appropriately choosing the metrics in the first place allows to avoid congestion in all cases, and for the "good" metrics the effect of all possible link failures is very similar, leading to a fairly predictable network behavior in case of failure. 


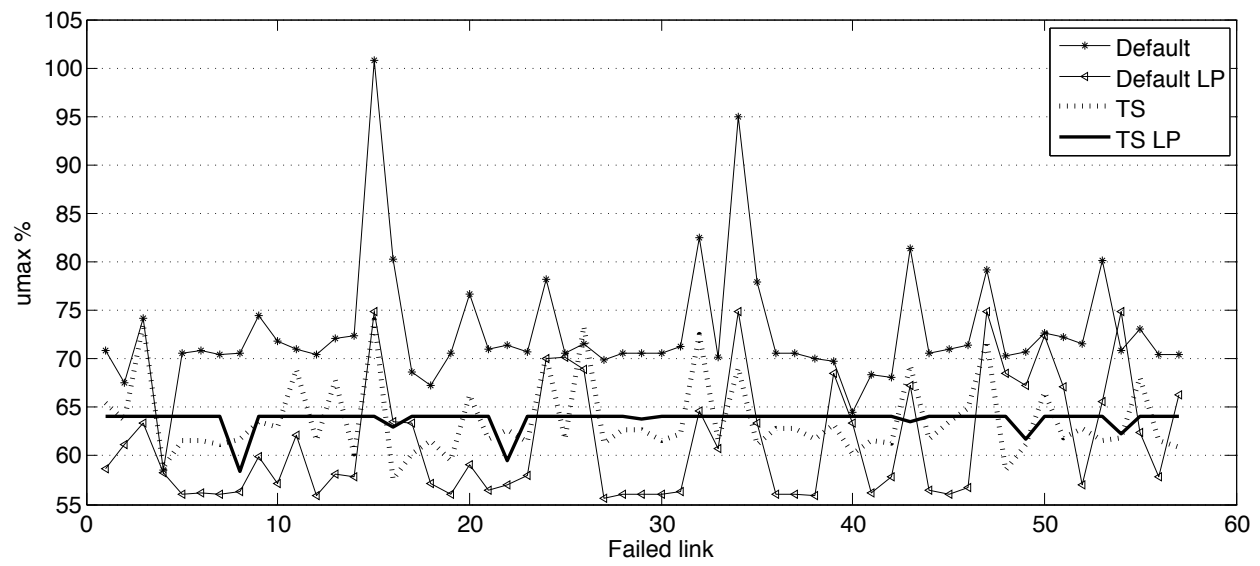

Figure 7: Objective function value in case of single link failure for IBCN network

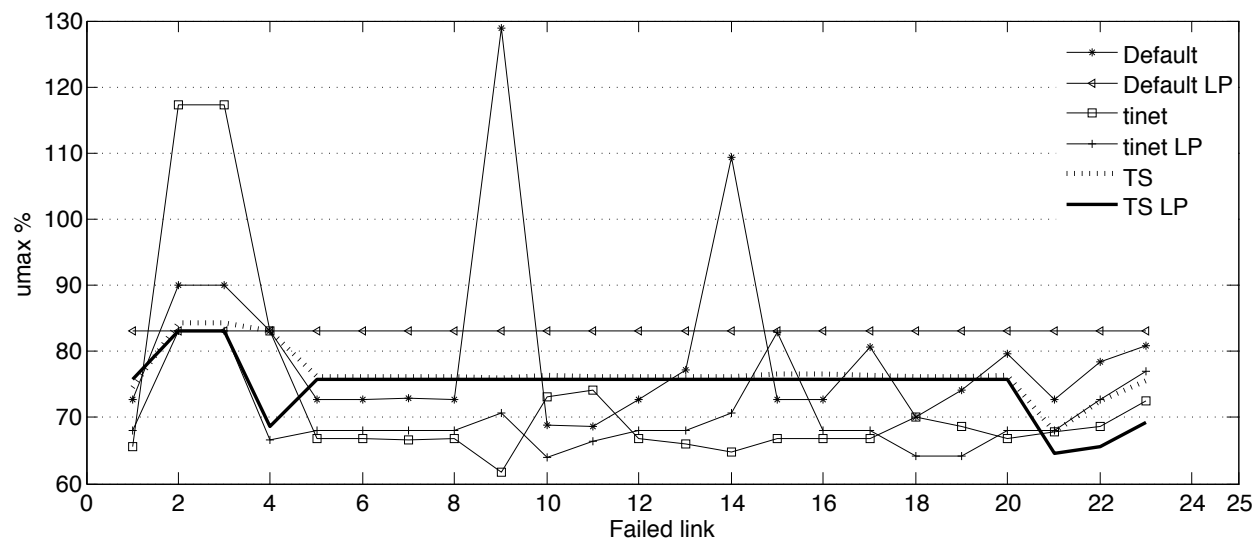

Figure 8: Objective function value in case of single link failure for Tinet network

\subsection{Impact of the traffic matrix}

As previously remarked, adjusting the IS-IS/OSPF weights can only be done infrequently, both for computational reasons and because it induces potentially large traffic shifts in the network, with potential service degradation. It may therefore be convenient to adjust the MPLS-TE LSPs only to react to changes in the traffic patterns. Thanks to the availability of different traffic matrices for the Géant network [61, we were able to test the behavior of this approach. The results are reported in Table 4 focussing only on the survivable model, for a sample of traffic matrices corresponding to the same day (23 ${ }^{\text {rd }}$ of April 2005) but to different hours of the day. Note that the metrics used in all of these simulations are the ones optimized by TS using as input the traffic matrix of 22:00 hours (the "worst case" ma- 


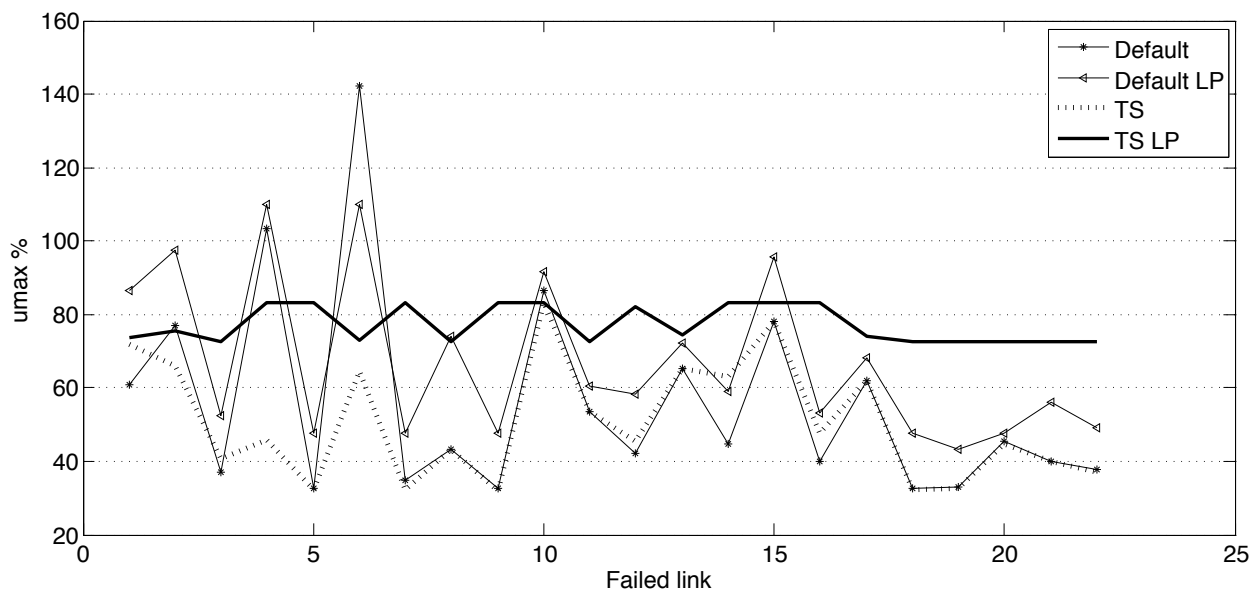

Figure 9: Objective function value in case of single link failure for Atlanta network

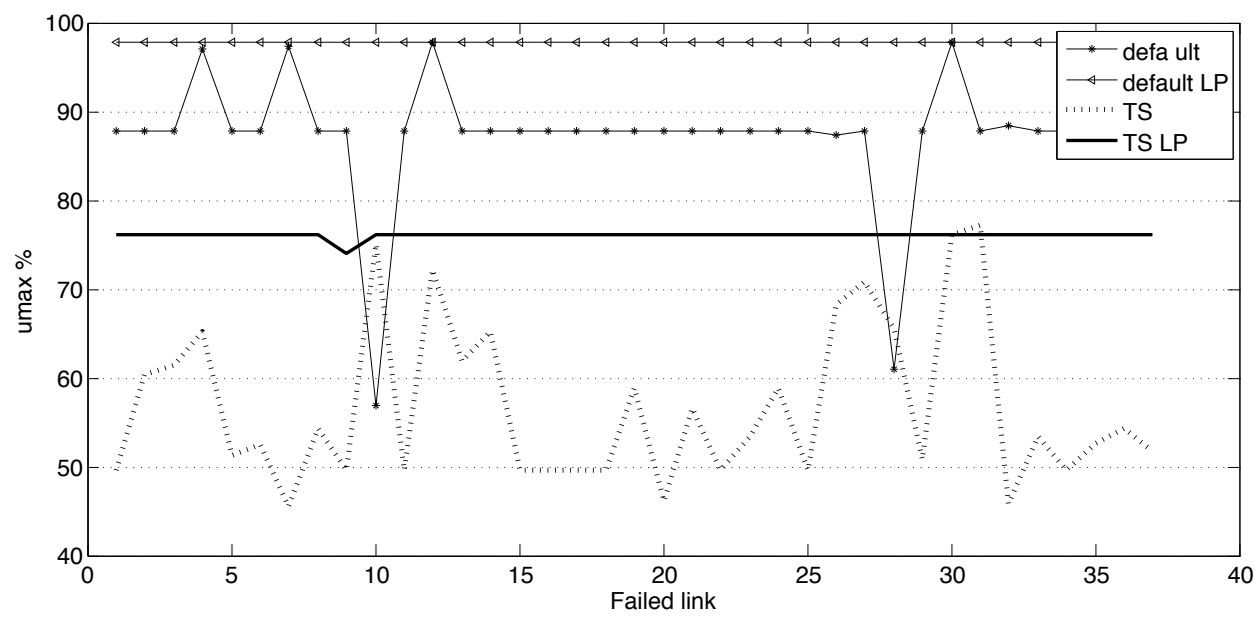

Figure 10: Objective function value in case of single link failure for Géant network

trix). The results show that in all cases but one, joint use of IS-IS/OSPF and MPLS-TE allows one to reduce the maximum link utilization. Moreover, the gain with respect to the IS-IS/OSPF routing alone is most often significant, being greater than $40 \%$ in one third of cases. Thus, the approach is reasonably able to cope with traffic matrices changing over time. More sophisticated versions of the models explicitly taking into account uncertainty of the traffic matrices can be developed with approaches akin those used in [6, 7, 9, 13, 17, 18, this will be the subject of future research. 
Table 4: Results for different traffic matrices of Géant Network

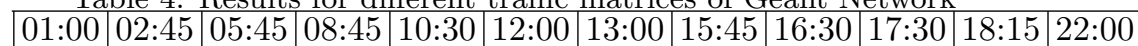

\begin{tabular}{|l|r|r|r|r|r|r|r|r|r|r|r|r|}
\hline OSPF & 74.03 & 92.36 & 69.75 & 71.88 & 54.61 & 50.76 & 54.61 & 54.89 & 50.26 & 68.10 & 61.89 & 77.16 \\
comb. & 70.34 & 64.97 & 42.22 & 39.27 & 32.43 & 33.25 & 32.43 & 52.71 & 50.26 & 57.72 & 57.57 & 76.14 \\
\hline \# LSP & 184 & 186 & 276 & 267 & 240 & 248 & 240 & 211 & 192 & 227 & 262 & 210 \\
\hline
\end{tabular}

\begin{tabular}{|c|c|c|c|c|c|c|}
\hline & $\Delta+t_{9} n+9$ & & & & & $2 C \Delta \mathrm{T}$ \\
\hline & default TS & default TS & default & Tinet & TS & default TS \\
\hline$\delta=0$ & 129147 & $276 \quad 210$ & 134 & 83 & 139 & 671585 \\
\hline$\delta>0$ & $121 \quad 116$ & $251 \quad 195$ & 126 & 91 & 133 & $664 \quad 575$ \\
\hline
\end{tabular}

\subsection{Impact of $\delta$}

Finally, we have computationally tested the idea of modifying the objective function of the models to (6), where the "small" parameter $\delta$ discourages flow to use MPLS-TE, in order to try to reduce the total number of LSPs to be set. Starting with $\delta=1 \mathrm{e}-6$, and decreasing it by one order of magnitude at each step, we found that $\delta=1 \mathrm{e}-14$ allows one to reduce the number of LSPs in the optimal solution in all but one of the tested instances, as reported in Table 5, without increasing the value of $u_{\max }$. Therefore, our experiments confirm that setting $\delta \neq 0$ in (6) can be an effective (and fast) way to reduce the number of LSPs in the solutions returned by the proposed approach.

\section{Conclusions}

This paper presents Linear Programming models for the problem of minimizing the maximum link utilization under the joint use of the IS-IS/OPSF routing protocol and of the MPLS-TE technology. To the best of our knowledge, this is the first exact approach proposed in the literature that embeds in a unique overall model the two different technologies while taking into account network survivability in failure conditions. By using an off-the-shelf open source LP solver, optimal configurations of the complementary MPLSTE LSPs is obtained in short time on a standard PC; by contrast, the time required for finding optimized IS-IS/OSPF metrics is much higher, reflecting the inherent difference in complexity between the two problems. The obtained results show that the proposed models can attain - at least in the tested instances - better levels of network utilization than those achievable via the more traditional IGP routing protocols. In fact, due to the static nature of the IGP routing algorithms, with IS-IS/OSPF protocols the traffic is mostly routed on the least cost paths, possibly causing congestion of some links while leaving other links lightly loaded. On the contrary, MPLS-TE 
can route the traffic through explicit paths, optimizing network resources utilization and traffic performance by distributing the flows over the whole network and reducing waste of resources. Hence, the models can be beneficial for Quality of Service routing of traffic with particular performance requirements. Moreover, survivability of the service delivery is guaranteed following the link restoration scheme. The model solution can be obtained in nearly real time even for real telecommunication networks; furthermore, it is possible to implement the results of the model with a very limited intervention of the network operators. Thus, the model can be actually applied in a real production environment, possibly yielding relevant economic savings because the better utilization of network resource may reduce and/or delay the investments required to upgrade the network. Finally, the model can provide a solid foundation for more sophisticated approaches, e.g. considering uncertainty in the traffic matrix and/or the possibility to upgrade network equipment.

Acknowledgements. This work has been partially supported by Tiscali S.p.A within the framework of the ICE Lab (Information and Communications Engineering Academy joint Laboratory). The authors would like to thank Mr. Paolo Susnik and Mr. Giorgio Lembo for supporting the work and the reviewers for their valuable comments that helped improve the manuscript.

\section{References}

[1] T. Crainic, A. Frangioni, B. Gendron, Multicommodity Capacitated Network Design, in: Soriano, P., Sanso, B. (Eds.), Telecommunications Network Planning, Kluwer Academics Publisher, 1999, pp. 1-19.

[2] M. Pióro, D. Medhi, Routing, Flow, and Capacity Design in Communication and Computer Networks, Morgan Kaufmann Publishers Inc., San Francisco, CA, USA, 2004.

[3] J. Lenstra, A. Rinnooy-Kan, On general routing problems, Networks 6 (1976) 273-280.

[4] B. Fortz, M. Thorup, Increasing internet capacity using local search, Computational Optimization and Applications 29 (2004) 13-48.

[5] D. Applegate, E. Cohen, Making intra-domain routing robust to changing and uncertain traffic demands: understanding fundamental tradeoffs, in: in Proc. SIGCOMM 03, ACM, NY, 2003, pp. 313-324. 
[6] W. Ben-Ameur, H. Kerivin, Routing of Uncertain Traffic Demands, Optimization and Engineering 6 (3) (2005) 283-313.

[7] W. Ben-Ameur, H. Kerivin, Networks new economical virtual private, Commun. ACM 46 (6) (2003) 69-73.

[8] A. Gupta, J. Kleinberg, A. Kumar, R. Rastogi, B. Yener, Provisioning a virtual private network: a network design problem for multicommodity flow, in: STOC '01: Proceedings of the thirty-third annual ACM symposium on Theory of computing, ACM, New York, NY, USA, 2001, pp. 389-398.

[9] C. Chekuri, F. Shepherd, G. Oriolo, M. Scutellà, Hardness of robust network design, Netw. 50 (1) (2007) 50-54.

[10] M. Scutellà, On improving optimal oblivious routing, Operations Research Letters 37 (3) (2009) 197-200.

[11] N. Goyal, N. Olver, F. Shepherd, The VPN conjecture is true, in: STOC '08: Proceedings of the 40th annual ACM symposium on Theory of computing, ACM, New York, NY, USA, 2008, pp. 443-450.

[12] M. Labbé and R. Séguin and P. Soriano and C. Wynants, Network Synthesis with Non-Simultaneous Multicommodity Flow Requirements: Bounds and Heuristics, Tech. rep. (1999).

[13] C. Chekuri, Routing and network design with robustness to changing or uncertain traffic demands, SIGACT News 38 (3) (2007) 106-129.

[14] C. Hurkens, J. Keijsper, L. Stougie, Virtual Private Network Design: A Proof of the Tree Routing Conjecture on Ring Networks, SIAM J. Discret. Math. 21 (2) (2007) 482-503.

[15] D. P. Goyal, N. G. Duffield, P. Goyal, A. Greenberg, P. Mishra, K. K. Ramakrishnan, J. E. V. D. Merwe, A Flexible Model for Resource Management in Virtual Private Networks, in: in Proc. ACM SIGCOMM, 1999, pp. 95-108.

[16] J. Fingerhut, S. Suri, J. Turner, Designing least-cost nonblocking broadband networks, J. Algorithms 24 (2) (1997) 287-309.

[17] P. Belotti, M. Pınar, Optimal oblivious routing under linear and ellipsoidal uncertainty, Optimization and Engineering 9 (3) (2008) 257-271. 
[18] D. Bertsimas, M. Sim, Robust discrete optimization and network flows, Mathematical Programming 98 (1) (2003) 49-71.

[19] A. Markopoulou and G. Iannaccone and S. Bhattacharyya and C. Chuah and Y. Ganjali and C. Diot, Characterization of failures in an operational IP backbone network, IEEE/ACM Trans. Netw. 16 (4) (2008) $749-762$.

[20] L. Li, M. Buddhikot, C. Chekuri, K. Guo, Routing bandwidth guaranteed paths with local restoration in label switched networks, IEEE Journal on Selected Areas in Communications 23 (2) (2005) 437-449.

[21] E. Yetginer and E. Karasan, Robust Path Design Algorithms for Traffic Engineering with Restoration in MPLS Networks, in: ISCC '02: Proceedings of the Seventh International Symposium on Computers and Communications (ISCC'02), IEEE Computer Society, 2002, p. 933.

[22] S. Beker and N. Puech and V. Friderikos, A Tabu Search Heuristic for the Offline MPLS Reduced Complexity Layout Design Problem, in: Networking, 2004, pp. 514-525.

[23] S. Cerav Erbas and C. Erbas, A Multiobjective Off-line Routing Model for MPLS Networks, in: Proc. of the 18th International Teletraffic Congress, 2003.

[24] A. Riedl, Optimized Routing Adaptation in IP Networks Utilizing OSPF and MPLS, in: IEEE 2003 International Conference on Communications (ICC), Anchorage, USA, May 2003, 2003.

[25] F. Skivée, S. Balon, G. Leduc, A Scalable Heuristic for Hybrid IP/MPLS Traffic Engineering - Case Study on an Operational Network, in: Proc. of IEEE International Conference on Networks (ICON), IEEE Press, 2006.

[26] S. Balon, J. Lepropre, O. Delcourt, F. Skivée and G. Leduc, Traffic Engineering an Operational Network with the TOTEM Toolbox, IEEE Transactions on Network and Service Management (TNSM) 4 (2007) $51-61$.

[27] L. S. Buriol, M. G. C. Resende and M. Thorup, Survivable IP network design with OSPF routing, Networks 49 (1) (2007) 51-64.

[28] B. Fortz, M. Thorup, Internet traffic engineering by optimizing OSPF weights, in: in Proc. IEEE INFOCOM, 2000, pp. 519-528. 
[29] B. Fortz and M. Thorup, Robust optimization of OSPF/IS-IS weights, in: In Proc. International Network Optimization Conference (INOC), 2003, pp. 225-230.

[30] M. Ericsson, M. Resende, P. Pardalos, A Genetic Algorithm for the weight setting problem in OSPF routing, Combinatorial Optimization 6 (2002) 299-333.

[31] E. Mulyana and U. Killat, A Hybrid Genetic Algorithm Approach for OSPF Weight Setting Problem, in: Peroc. of the $2^{\text {nd }}$ Polish-German Teletraffic Symposium PGTS, 2004.

[32] L.S. Buriol, M.G.C. Resende, C.C. Ribeiro and M.Thorup, A memetic algorithm for OSPF routing, in: Proc. of the $6^{\text {th }}$ INFORMS Telecom, 2002, pp. 187-188.

[33] J. Harmatos, A heuristic algorithm for solving the static weight assignment optimisation problem in OSPF networks, in: Proc. of of Global Internet Conference, 2002.

[34] M. Pióro, Á. Szentesi, J. Harmatos, A. Jüttner, P. Gajowniczek, S. Kozdrowski, On Open Shortest Path First related network optimisation problems, Perform. Eval. 48 (1/4) (2002) 201-223.

[35] A. Elwalid, C. Jin, S. H. Low and I. Widjaja, MATE: MPLS adaptive traffic engineering, in: INFOCOM, 2001, pp. 1300-1309.

[36] A. Elwalid and C. Jin and S. Low and I. Widjaja, Multi-Path Adaptive Traffic Engineering, Computer Networks 40 (2002) 695-709.

[37] M. S. Kodialam, T. V. Lakshman, Minimum Interference Routing with Applications to MPLS Traffic Engineering, in: INFOCOM'00, 2000, pp. 884-893.

[38] P. Aukia, M. Kodialam, P. Koppol, T. Lakshman, H. Sarin, B. Suter, RATES: a server for MPLS traffic engineering, Network, IEEE 14 (2) (2000) $34-41$.

[39] A. B. Bagula, Hybrid routing in next generation IP networks, Computer Communications 29 (2006) 879-892.

[40] S. Balon, G. Leduc, Combined intra- and inter-domain traffic engineering using hot-potato aware link weights optimization, in: SIGMETRICS, 2008, pp. 441-442. 
[41] M. Zagozdzon, M. Dzida and M. Pióro, Traffic Flow Optimization in Networks with Combined OSPF/MPLS Routing, in: ADCOM 2007, 2007, pp. 131-137.

[42] W. Ben-Ameur, N. Michel, B. Liau, E. Gourdin, Routing strategies for IP networks, Telektronikk (2001) 145-158.

[43] E. Mulyana, U. Killat, Optimization of IP networks in various hybrid IGP/MPLS routing schemes, in: MMB, 2004, pp. 295-304.

[44] M. Zhang, B. Liu, B. Zhang, Multi-Commodity Flow Traffic Engineering with hybrid MPLS/OSPF routing, in: Global Telecommunications Conference, 2009. GLOBECOM 2009. IEEE, 2009, pp. 1 -6.

[45] A. Nucci, B. Schroeder, S. Bhattacharrya, N. Taft, C. Diot, IGP Link Weight Assignment for Transient Link Failures, in: $18^{\text {th }}$ International Teletraffic Congress, Berlin, Germany, 2003.

[46] D. Yuan, A Bi-criteria Optimization Approach for Robust OSPF Routing, in: $6^{\text {th }}$ IEEE Workshop on IP Operation Management (IPOM), 2003.

[47] D. Cherubini, A. Fanni and C. Murgia, IS-IS/OSPF weights optimization for survivable backbone networks: a Tabu Search metaheuristic approach, in: OPNETWORK 2006, Washington DC, 2006.

[48] Y. Liu, D. Tipper, Spare capacity allocation for non-linear cost and failure-dependent path restoration, in: Proceeding of the $3^{\text {rd }}$ International Workshop on Design of Reliable Communication Networks, DRCN, 2001.

[49] R. Ahuja, T. Magnanti, J. Orlin, Network Flows: Theory, Algorithms, and Applications, Prentice-Hall, Inc., Upper Saddle River, NJ, USA, 1993.

[50] A. Mereu, D. Cherubini, A. Fanni, A. Frangioni, Primary and backup paths optimal design for traffic engineering in hybrid IGP/MPLS networks, in: Design of Reliable Communication Networks, 2009. DRCN 2009. 7th International Workshop on, 2009, pp. $273-280$.

[51] S. Balon, F. Skivée, G. Leduc, Comparing traffic engineering objective functions, in: CoNEXT '05: Proceedings of the 2005 ACM conference on Emerging network experiment and technology, ACM, 2005, pp. 224225 . 
[52] K. Jones, I. Lustig, J. Farwolden, W. Powell, Multicommodity Network Flows: The Impact of Formulation on Decomposition, Mathematical Programming 62 (1993) 95-117.

[53] A. Frangioni, G. Gallo, A Bundle Type Dual-Ascent Approach to Linear Multicommodity Min Cost Flow Problems, INFORMS Journal on Computing 11 (4) (1999) 370-393.

[54] B. Vatinlen, F. Chauvet, P. Chretienne, P. Mahey, Simple bounds and greedy algorithms for decomposing a flow into a minimal set of paths, European Journal of Operational Research 185 (3) (2008) 1390-1401.

[55] OsiSolver: http://www.coin-or.org.

[56] OPNET: http://www.opnet.com.

[57] ZIB - SNDlib: http://sndlib.zib.de/home.action.

[58] INTEC Broadband Communication Networks research group (IBCN). URL www . ibcn. intec.ugent. be

[59] Tiscali International Network (now Tinet) network map. URL www.tinet.net/network/network-map

[60] Géant Project: http://www.geant.net.

[61] S. Uhlig, B. Quoitin, J. Lepropre, S. Balon, Providing public intradomain traffic matrices to the research community, ACM SIGCOMM Computer Communication Review 36 (1) (2006) 83-86. 